\title{
MAIL TRANSFER PROTOCOI
}

\author{
Suzanne Sluizer \\ and \\ Jonathan B. Postel
}

RFC 780

May 1981

\begin{abstract}
Information Sciences Institute University of Southern California 4676 Admiralty Way

Marina del Rey, California 90291

(213) 822-1511
\end{abstract}



Mail Transfer Protocol

TABLE OF CONTENTS

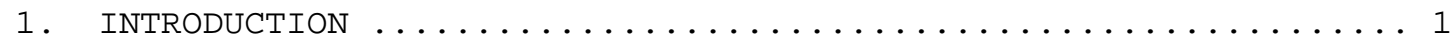

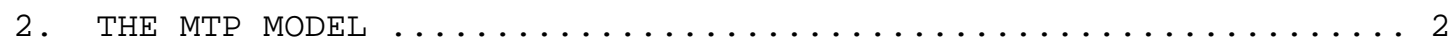

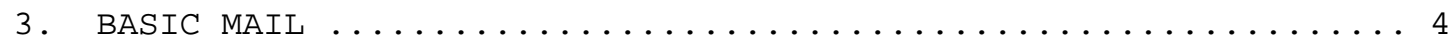

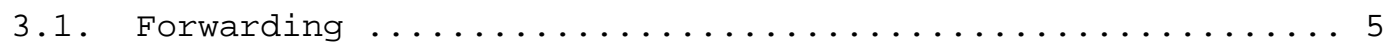

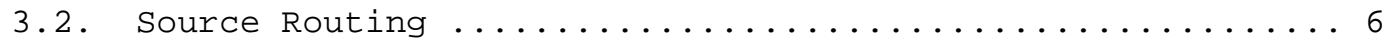

4. MULTI-RECIPIENT MAIL $\ldots \ldots \ldots \ldots \ldots \ldots \ldots \ldots \ldots \ldots \ldots \ldots \ldots \ldots \ldots \ldots$

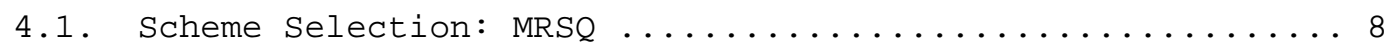

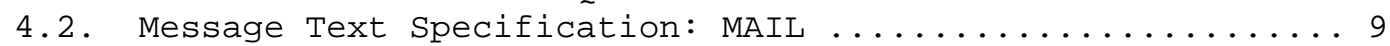

4.3. Recipient Specification: MRCP .................. 10

4.4. Scheme Mechanics: Recipients First ................ 10

4.5. Scheme Mechanics: Text First ................... 12

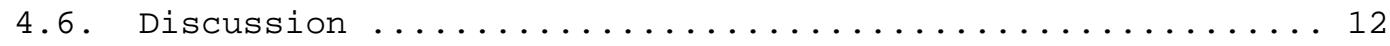

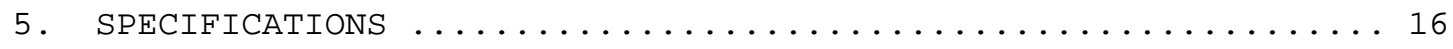

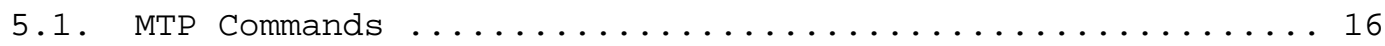

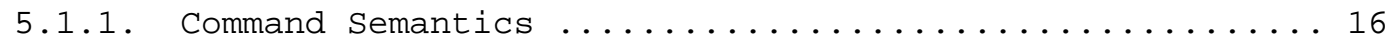

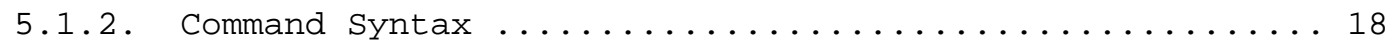

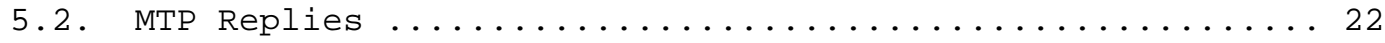

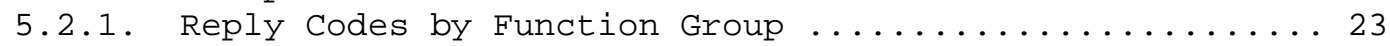

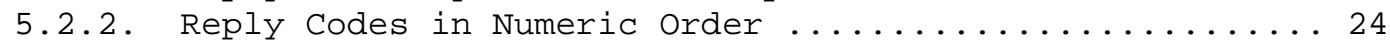

5.3. Sequencing of Commands and Replies .............. 25

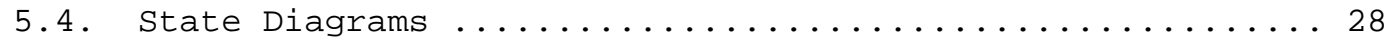

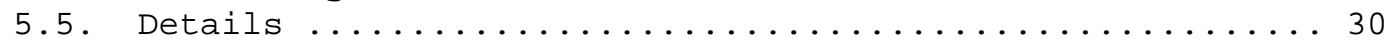

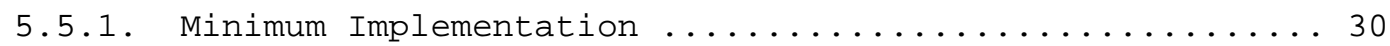

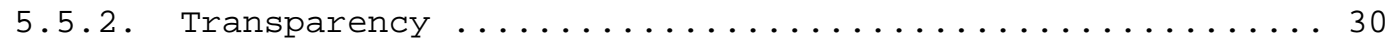

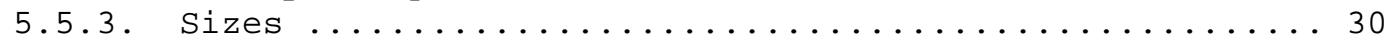

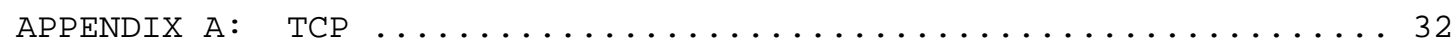

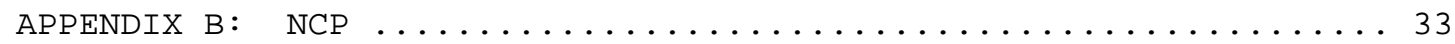

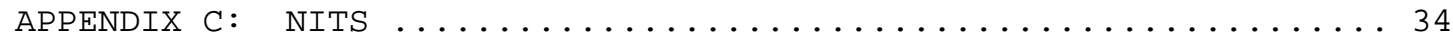

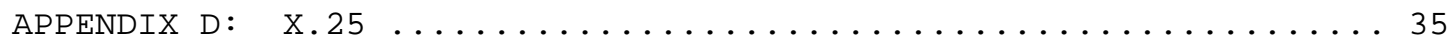

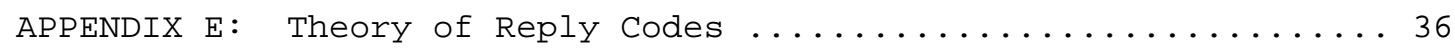

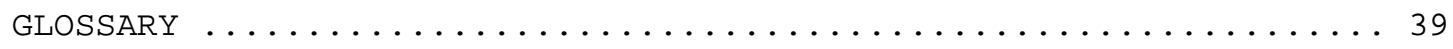

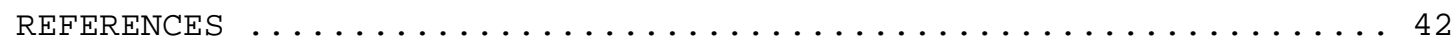



Network Working Group

Request for Comments: 780

Replaces: RFC 772
S. Sluizer

J. Postel

ISI

May 1981

MAIL TRANSFER PROTOCOL

\section{INTRODUCTION}

The objective of Mail Transfer Protocol (MTP) is to transfer mail reliably and efficiently.

MTP is designed to be independent of the particular transmission subsystem and requires only a reliable ordered data stream channel. Appendices describe the use of MTP with various transport services. A Glossary provides the definitions of terms as used in this document.

An important feature of MTP is its capability to relay mail from one transport environment to another. A transport service provides an interprocess communication environment (IPCE). An IPCE may cover one network, several networks, or a subset of a network. A process can communicate directly with another process anywhere in its own IPCE. Mail is a special case of interprocess communication. Mail can be communicated between proceses in different IPCEs by relaying through a process connected to two (or more) IPCEs. More specifically, mail can be relayed between hosts on different transport systems by a host on both transport systems. It is important to realize that transport systems (or IPCEs) are not one-to-one with networks. 


\section{THE MTP MODEL}

The MTP design is based on the following model of communication: at the initiation of the user, the sender-MTP establishes the full-duplex transmission channel. MTP commands are generated by the sender-MTP and sent to the receiver-MTP. MTP replies are sent from the receiver-MTP to the sender-MTP in response to the commands.

In the simplest case, once the transmission channel is established the MTP-sender sends a MAIL command indicating the sender and receiver of the mail. If the MTP-receiver can accept the mail it responds with a go ahead reply. Then the MTP-sender sends the mail data, terminating with a special sequence. If the MTP-receiver successfully processes the mail it responds with an ok reply.

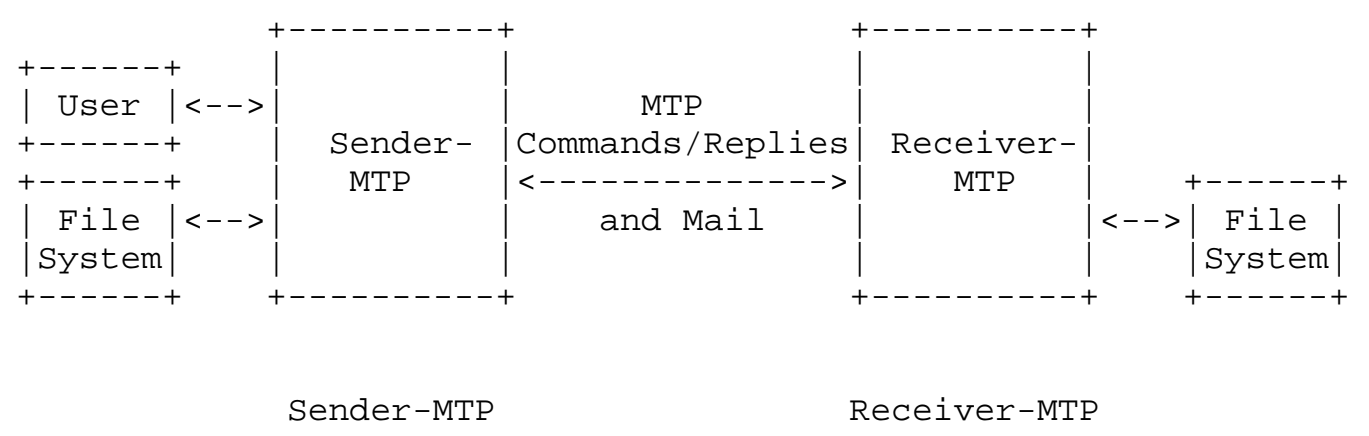

Model for MTP Use

Figure 1

The MTP provides mechanisms for the transmission of mail; directly from the sending user's host to the receiving user's host when the two host are connected to the same transport service, or via one or more relay MTP-servers when the source and destination hosts are not connected to the same transport service.

To be able to provide the relay capability the MTP-server must be supplied with the name of the ultimate destination host as well as the destination mailbox name. 
The arguments to the MAIL command are a FROM path and a TO path. The TO path is a source route while the FROM path is a return route (which may be used to return a message to the sender when an error occurs with a relayed message).

The preceding discussion has outlined the transmission of one copy of one message from a source to a destination host and the possibility of relaying messages between different transport services. The MTP additionally supports the transmission of one copy of a message addressed to multiple recipients.

In order for mail to be successfully transmitted the destination users must be known at the destination receiver-MTP and the mail data must be correctly received and stored. In the single recipient case discussed above the positive response to the MAIL command indicated the recipient was known, and the final ok response indicated the mail was received and stored.

To support multi-recipient mail, MTP provides two procedures: Text-First, and Recipients-First. In the text-first scheme the mail data is sent and acknowledged, then each recipient identification is sent and acknowledged (or refused) separately. In the recipients-first scheme the recipients are negotiated first, then the text is sent and acknowledged (for all recipients at once). The choice of scheme is up to the MTP-receiver, and depends on the way mail is handled in the destination host.

The multi-recipient mail procedures are optional and the determination of which scheme to use is negotiated. The use of the multi-recipient schemes is strongly encouraged by the economy they provide in transmission and processing.

The mail commands and replies have a rigid syntax. Replies also have a numeric code. In the following, examples appear which use actual commands and replies. The complete lists of commands and replies appears in section 5 on specifications.

Commands and replies are not case sensitive. That is, a command or reply word may be upper case, lower case, or any mixture of upper and lower case. Note that this is not true of mailbox user names. For some hosts the user name is case sensitive, and MTP implementations must take case to preserve the case of user names as they appear in mailbox arguments. 


\section{BASIC MAIL}

The basic command for transmitting mail is MAIL. This command causes the transmitted data to be entered into the recipient's mailbox, or accepted for relaying to the destination host.

The mail text is also sent on the transmission channel. This requires that the end of the text be signalled so that the command and reply dialog can be resumed. MTP signals the end of the mail text by sending a line containing only a period. A transparency procedure is used to prevent this interfering with the users text (see Section 5.5.2).

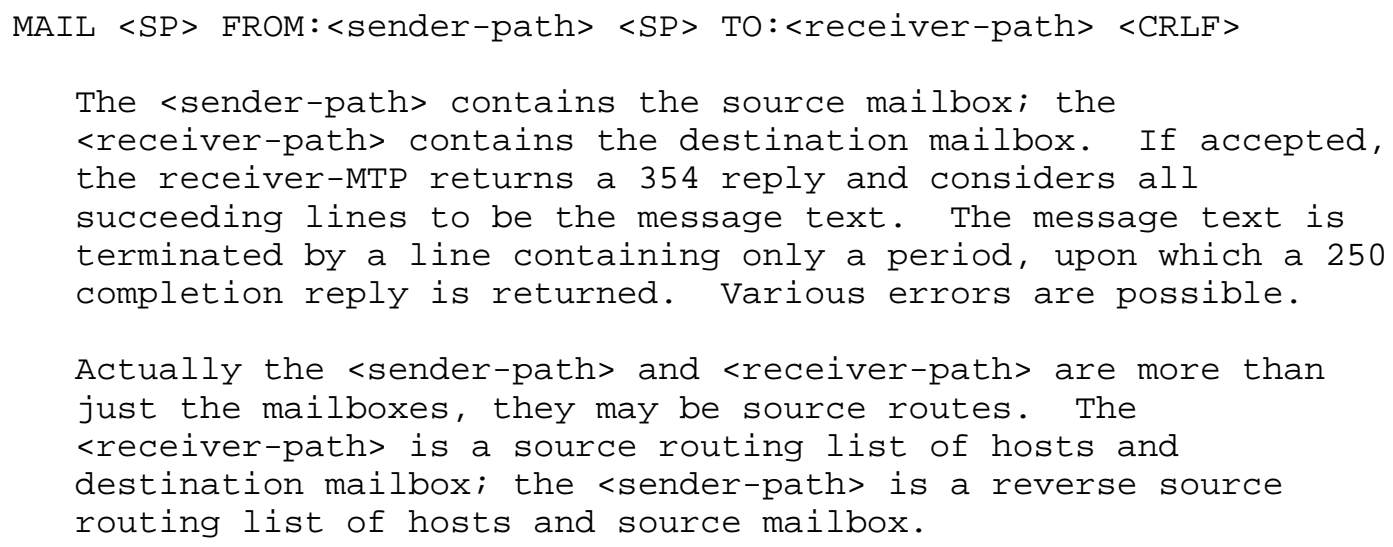




\section{Example of MAIL (Basic Mail)}

This MAIL command specifies the mail is sent by Waldo at host A, and is to be delivered to Foo at host $Y$. Here we assume that host A contacts host $Y$ directly.

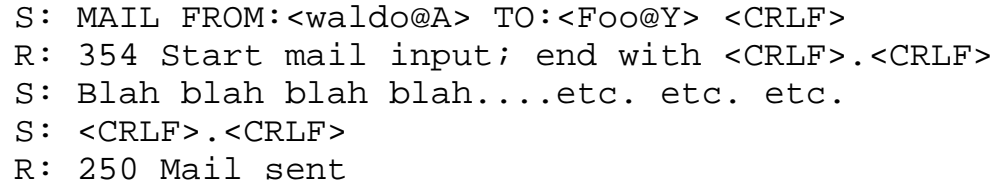

The mail text has now been sent to "Foo".

Example 1

\subsection{FORWARDING}

There are two possible preliminary replies that a receiver may use to indicate that it is accepting mail for a user whose mailbox is not at that host.

151 User not local; will forward to <user>e<host>

This reply indicates that the receiver-MTP knows the user's mailbox is on another host and will take responsibility for forwarding the mail to that host. This reply is only sent when the sender would not expect the mail to be forwarded. That is, when <receiver-path> as given in the command indicates mail relaying, this reply will not be used. This reply could be used for an organization with several hosts when each has a list of many of the users on the hosts. A host can accept mail for any user on its list and forward it to the correct host.

152 User Unknown; mail will be forwarded by the operator

This reply indicates that the host does not recognize the user name, but that it will accept the mail and have the operator attempt to deliver it. This is useful if the user name is misspelled, but may be a disservice if the mail is really undeliverable. 
If forwarding by the operator is unacceptable or if the sending-user would prefer to send the mail directly to the recipient's actual host, the action may be aborted.

The MTP-sender must accept or reject the proposal in the preliminary reply by sending a continue (CONT) or abort (ABRT) command. In the case of the continue, the next reply from the MTP-receiver will be any of the replies expected for the MAIL command, most likely "354 Start mail input, ...". In the case of the abort, the next reply from the MTP-receiver will be "201 command okay, action aborted".

\subsection{SOURCE ROUTING}

The receiver-path may be a source route of the form "@ONE, @TWO, JOE@THREE", where ONE, TWO, and THREE are hosts. This form is used to emphasize the distinction between an address and a route.

At some distant future time it might be necessary to expand the mailbox format to include a region identifier, such as "user@hosteregion". If this occured the MTP path convention could be expanded to

"hosteregion, host@region, ...user@hosteregion". For example, "ONE@R1, TWO@R2, JOE@THREE@R3" .

The mailbox is an absolute address, and the route is information about how to get there. The two concepts should not be confused.

The elements of the receiver-path are to be moved to the sender-path as the message is relayed from one MTP to another. The sender-path is a reverse source route, that is, a source route to the originator of the message. When an MTP deletes its identifier from the receiver-path and inserts it into the sender-path, it must use the name it is known by in the environment it is sending into, not the environment the mail came from, in case the MTP is known be different names in different environments.

When source routing is used the receiver-MTP will receive mail to be relayed to another MTP. The receiver-MTP may accept the task of relaying the mail or reject it in the same way it accepts or reject mail for a local user. It does not use the 151 "User not local" or 152 "User unknown" preliminary replies. Once the receiver-MTP accepts the relaying task it receives the mail text and transforms the command arguments by removing its own identifier from the receiver-path and inserting it in the 


\begin{abstract}
beginning of the sender-path. The receiver-MTP then becomes a sender-MTP and establishes a transmission channel to the next MTP in the receiver-path and sends it the mail.

If an MTP has accepted the task of relaying the mail and later finds that the receiver-path is incorrect or that the mail cannot be delivered for whatever reason, then it must construct a notification message and send it to the originator of the undeliverable mail as indicated by the sender-path. This notification message must be from the MTP at this host. That is, the sender-path of the notification message itself will be "MTPQ<host>", and in the notification message header the From field will be "MTP at <host>". Of course, MTPs should not send notification messages about problems with notification messages.
\end{abstract}




\section{MULTI-RECIPIENT MAIL}

There are two MTP commands which allow the text of a message to be mailed to several recipients simultaneously; such message transmission is far more efficient than the practice of sending the text again and again for each additional recipient at a host. In one scheme, all recipients are specified first, and then the text is sent. In the other scheme, the order is reversed and the text is sent first, followed by the recipients. The sender-MTP suggests the scheme it would prefer, but receiver-MTP controls which scheme is actually used. To select a particular scheme, the MRSQ command is used; to specify recipients after a scheme is chosen, MRCP commands are given; and to furnish text, the MAIL command is used.

Both schemes are necessary because neither by itself is optimal for all systems. MRSQ R allows more of a "bulk" mailing because everything is saved up and then mailed simultaneously. This is very useful for systems such as ITS where the MTP-receiver does not itself write mail directly, but hands it on to a central mailer demon. The more information (e.g., recipients) associated with a single "hand-off", the more efficiently mail can be delivered.

By contrast, MRSQ $\mathrm{T}$ is geared to receiver-MTPs which want to deliver mail directly, in one-by-one incremental fashion. For each given recipient this scheme returns an individual success/failure reply code which may depend on variable mail system factors such as exceeding disk allocation, mailbox access conflicts, and so forth. If these receiver-MTPs tried to emulate MRSQ Rs bulk mailing, they would have to ensure that a success reply to the MAIL indeed meant that it had been delivered to ALL recipients specified -- not just some.

\subsection{SCHEME SELECTION: MRSQ}

MRSQ is the means by which a sender-MTP can test for MRSQ/MRCP implementation, select a particular scheme, reset its state, and even do some rudimentary negotiation. Its format is as follows:

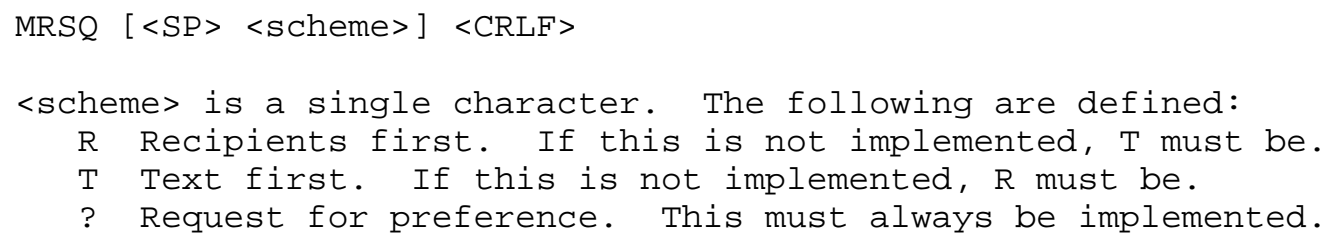




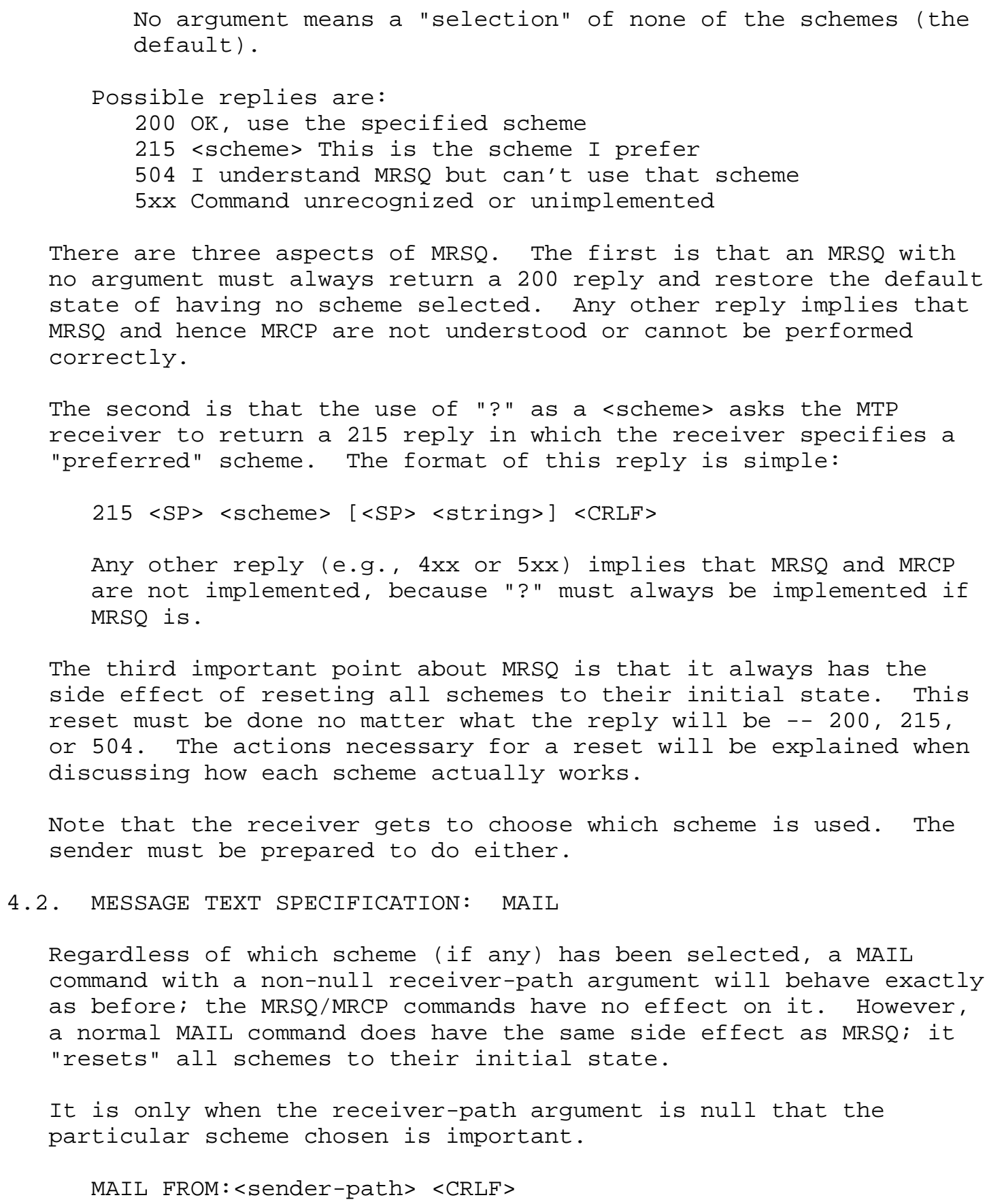

\subsection{MESSAGE TEXT SPECIFICATION: MAIL}

Regardless of which scheme (if any) has been selected, a MAIL command with a non-null receiver-path argument will behave exactly as before; the MRSQ/MRCP commands have no effect on it. However, a normal MAIL command does have the same side effect as MRSQ; it "resets" all schemes to their initial state.

It is only when the receiver-path argument is null that the particular scheme chosen is important.

MAIL FROM: $<$ sender-path $>\langle$ CRLF $>$ 
Rather than producing an error, the receiver will accept message text for this "null" recipient specification. What it does with it depends on which scheme is in effect, and will be described in the section on Scheme Mechanics.

\subsection{RECIPIENT SPECIFICATION: MRCP}

In order to specify recipient names (i.e., mailboxes) and receive some acknowledgment (or refusal) for each name, the following command is used:

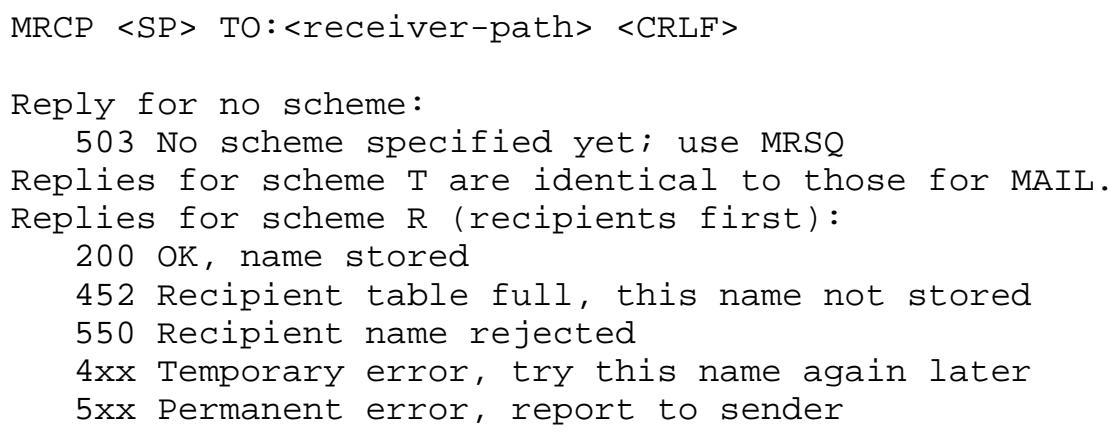

Note that use of this command is an error if no scheme has been selected yet; an MRSQ <scheme> must have been given if MRCP is to be used.

\subsection{SCHEME MECHANICS: MRSQ R (RECIPIENTS-FIRST)}

In the recipients-first scheme, MRCP is used to specify names which the MTP receiver stores in a list or table. Normally the reply for each MRCP will be either a 200 for acceptance or a $4 \mathrm{xx} / 5 \mathrm{xx}$ rejection code. All $5 \mathrm{xx}$ codes are permanent rejections (e.g., user not known) which should be reported to the human user, whereas $4 \mathrm{xx}$ codes in general connote some temporary error that may be rectified later. None of the $4 \mathrm{xx} / 5 \mathrm{xx}$ replies impinge on previous or succeeding MRCP commands, except for 452 which indicates that no further MRCPs will succeed unless a message is sent to the already stored recipients or a reset is done.

Sending message text to stored recipients is done by giving a MAIL command with no receiver-path argument; that is, just MAIL <SP> FROM: <sender-path> <CRLF>. Transmission of the message text is exactly the same as for normal MAIL. However, a positive acknowledgment at the end of transmission means the message has been sent to ALL recipients that were remembered with MRCP, and a 
failure code means that it should be considered to have failed for ALL of these specified recipients. This applies regardless of the actual error code. Regardless of what the reply signifies, all stored recipient names are flushed and forgotten -- in other words, things are reset to their initial state. This purging of the recipient name list must also be done as the reset side effect of any use of MRSQ (or MAIL with a non-null receiver-path argument).

A 452 reply (out of storage space) to an MRCP can be handled by using MAIL to specify the message for currently stored recipients, and then sending more MRCPs and another MAIL, as many times as necessary. For example, if a receiver only had room for 10 names this would result in a 50-recipient message being sent 5 times, to 10 different recipients each time.

If a sender attempts to specify message text (MAIL with no receiver-path argument) before any successful MRCPs have been given, this should be treated exactly as a "normal" MAIL with a null recipient would be; some receivers return an error, such as "550 Null recipient".

\section{Example of MRSQ R (Recipients First)}

First the sender must establish that the receiver implements MRSQ .

S: $\mathrm{MRSQ}<\mathrm{CRLF}>$

$\mathrm{R}: 200 \mathrm{OK}$, no scheme selected

An MRSQ with a null argument always returns a 200 if implemented, selecting the default "scheme", i.e., none of them. If MRSQ were not implemented, a code of $4 \mathrm{xx}$ or $5 \mathrm{xx}$ would be returned.

S: MRSQ $\mathrm{R}<\mathrm{CRLF}>$

$R: 200 \mathrm{OK}$, using that scheme

All is well; now the recipients can be specified.

S: MRCP TO: $\angle$ FOO@Y $><$ CRLF $>$

$\mathrm{R}: 200 \mathrm{OK}$ 


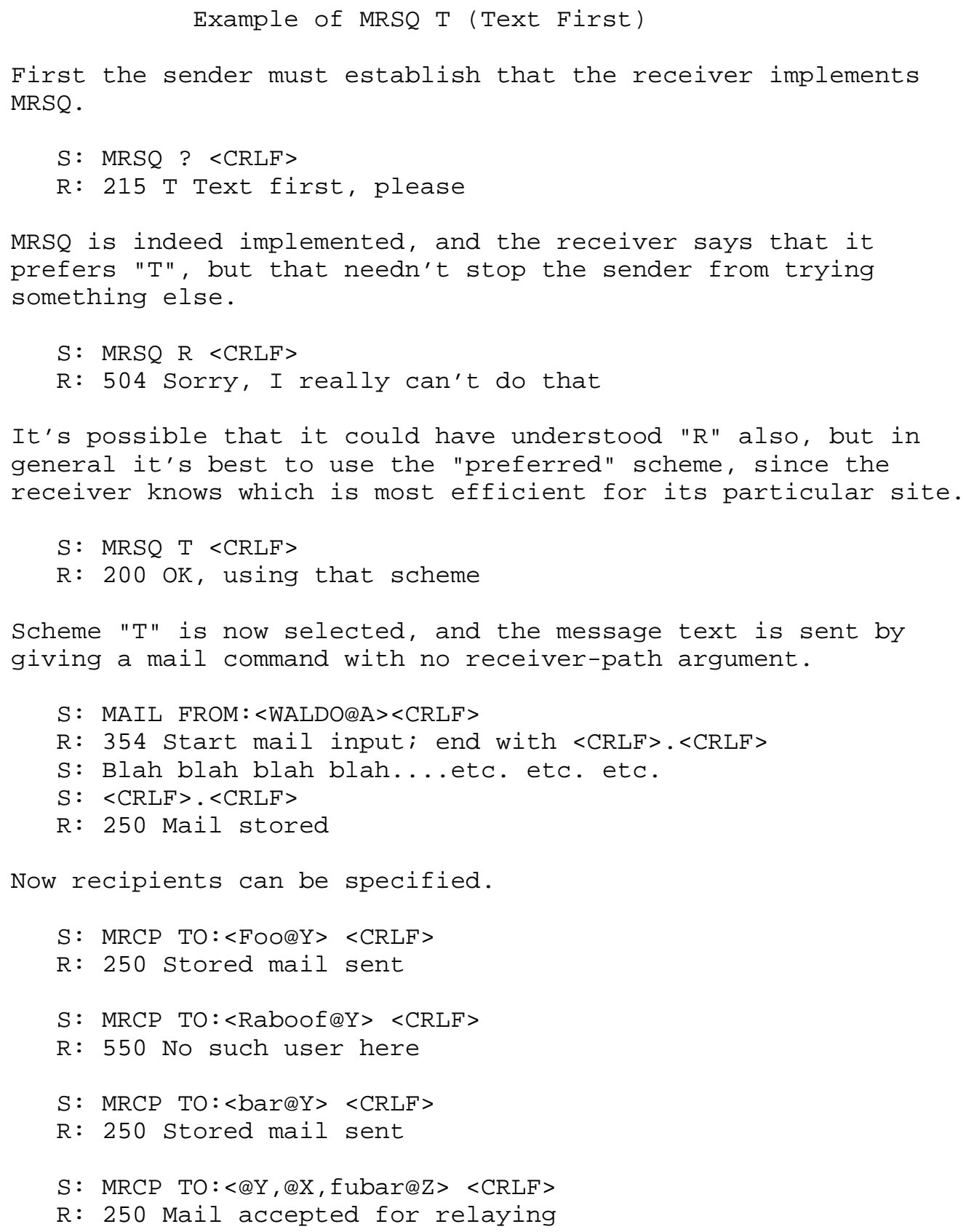


The text has now been sent to "Foo" and "bar" at host "Y" and will be relayed to "fubarez" through host "X", and still remains stored. A new message can be sent with another MAIL/MRCP ... sequence, but a careful sender would reset the state using the exchange below.

S: MRSQ ? <CRLF>

$R: 215 \mathrm{~T}$ Text first, please

Which resets the state without altering the scheme in effect.

Example 3

\subsection{DISCUSSION}

Because these commands are not required in the minimum implementation of MTP, one must be prepared to deal with sites which don't recognize either MRSQ or MRCP. "MRSQ" and "MRSQ ?" are explicitly designed as tests to see whether either scheme is implemented. MRCP is not designed as a test, and a failure return of the "unimplemented" variety could be confused with "No scheme selected yet", or even with "Recipient unknown".

There is no way to indicate in a positive response to "MRSQ ?" that the preferred "scheme" for a receiver is that of the default state; i.e., none of the multi-recipient schemes. The rationale is that in this case, it would be pointless to implement MRSQ/MRCP at all, and the response would therefore be negative.

One reason that the use of MAIL is restricted to null receiver-path arguments with this multi-recipient extension is the ambiguity that would result if a non-null receiver-path argument were allowed. For example, if MRSQ $R$ was in effect and some MRCPs had been given, and a MAIL FROM: $\mathrm{XQY}>\mathrm{TO}:\langle$ FOOQZ $>\langle\mathrm{CRLF}\rangle$ was done, there would be no way to distinguish a failure reply for mailbox "FOO" from a global failure for all recipients specified. A similar situation exists for MRSQ $T$; it would not be clear whether the text was stored and the mailbox failed, or vice versa, or both.

\footnotetext{
"Resets" of all schemes are done by all MRSQs and "normal" MAILs to avoid confusion and overly complicated implementation. The MRSQ command implies a change or uncertainty of status, and the MAIL command would otherwise have to use some independent
} 
mechanisms to avoid clobbering the data bases (e.g., message text storage area) used by the $T / R$ schemes. However, once a scheme is selected, it remains in effect. The recommended way for doing a reset, without changing the current selection, is with "MRSQ ?". Remember that "MRSQ" alone reverts to the no-scheme state. 


\section{SPECIFICATIONS}

\subsection{MTP COMMANDS}

\subsubsection{COMMAND SEMANTICS}

The MTP commands define the mail transfer or the mail system function requested by the user. MTP commands are character strings terminated by $\langle\mathrm{CRLF}>$. The command codes themselves are alphabetic characters terminated by $\langle$ SP $\rangle$ if parameters follow and <CRLF> otherwise. The syntax of mailboxes must conform to receiver site conventions. The MTP commands are discussed below. MTP replies are discussed in the section 5.2.

\section{MAIL (MAIL)}

This command is used to send mail over the transmission channel. The argument field contains a sender-path sequence and optional receiver-path sequence.

The sender-path sequence consists of an optional list of hosts and the sender mailbox. When the list of hosts is present, it is "reverse" source routing information and indicates that the mail was relayed through each host on the list (the first host in the list was the most recent relay). This list is used as source routing to return non-delivery notices to the sender. As each relay host adds itself to the beginning of the list, it must use its name as known in the network to which it is relaying the mail rather than the network from which the mail came (if they are different).

If the receiver-path sequence is present, it consists of an optional list of hosts and a destination mailbox. When the list of hosts is present, it is source routing information and indicates that the mail must be relayed to the first host on the list.

The receiver treats the lines following the command as mail text from the sender. The mail text is terminated by the character sequence "<CRLF>. $\angle \mathrm{CRLF}>$ ", (see Section 5.5.2 on Transparency).

As mail is relayed along the receiver-path sequence, each relay host must remove itself from the path sequence and put itself at the beginning of the sender-path sequence. When mail reaches its ultimate destination (the receiver-path 


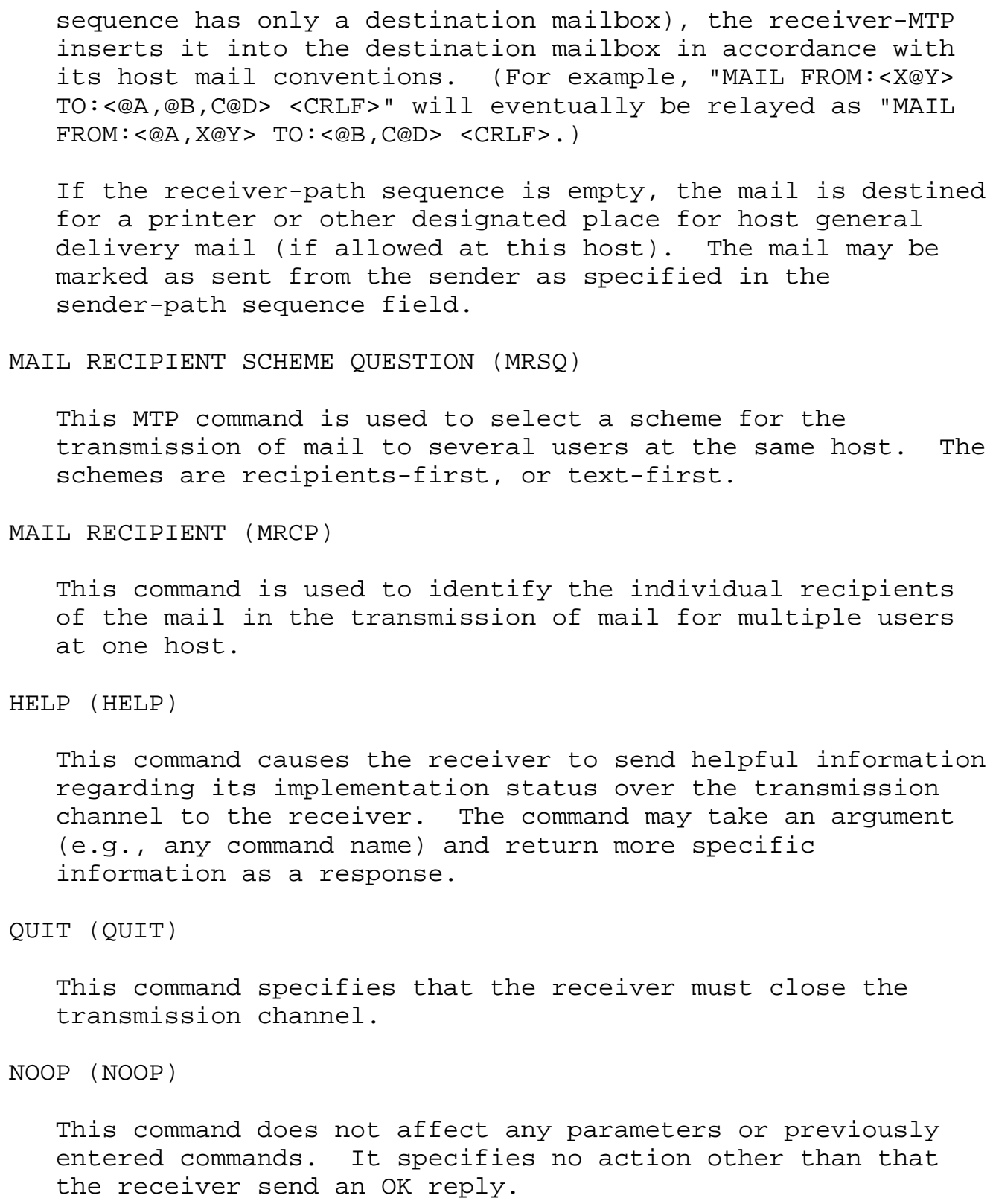


CONTINUE (CONT)

This command specifies that the previously specified action is to be continued. This is sent only following a preliminary reply.

ABORT (ABRT)

This command specifies that the previously specified action is to be aborted. This is sent only following a preliminary reply. It specifies no further action other than that the receiver send an oK reply.

\subsubsection{COMMAND SYNTAX}

The commands begin with a command code followed by an argument field. The command codes are four alphabetic characters. Upper and lower case alphabetic characters are to be treated identically. Thus any of the following may represent the mail command:

$$
\text { MAIL Mail mail MaIl mAIl }
$$

This also applies to any symbols representing parameter values, such as $\mathrm{R}$ or $r$ for RECIPIENT first. The command codes and the argument fields are separated by one or more spaces.

But, note that in the sender-path and receiver-path arguments case is important. In particular, in some hosts the user "foo" is different from the user "Foo".

The argument field consists of a variable length character string ending with the character sequence <CRLF>. It should be noted that the receiver is to take no action until the end of the line is received.

Square brackets denote an optional argument field. If the option is not taken, the appropriate default is implied. All characters are in the ASCII characters set. 


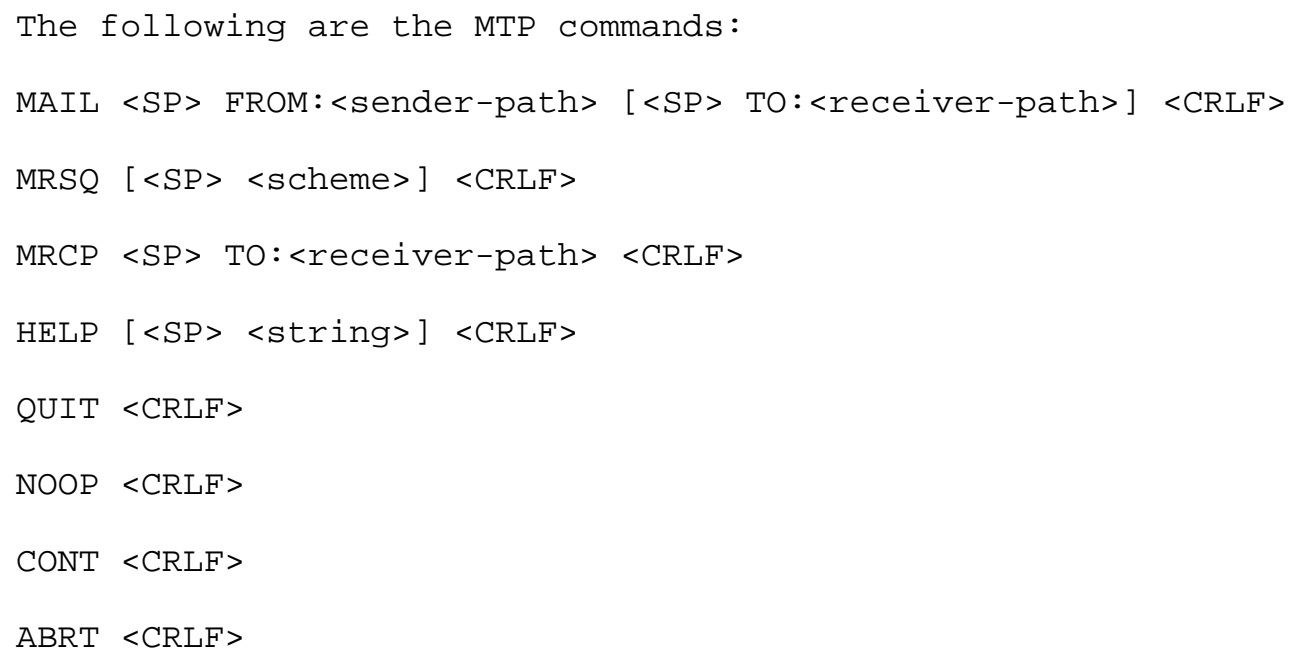


The syntax of the above argument fields (using BNF notation where applicable) is given below. The "..." notation indicates that a field may be repeated one or more times.

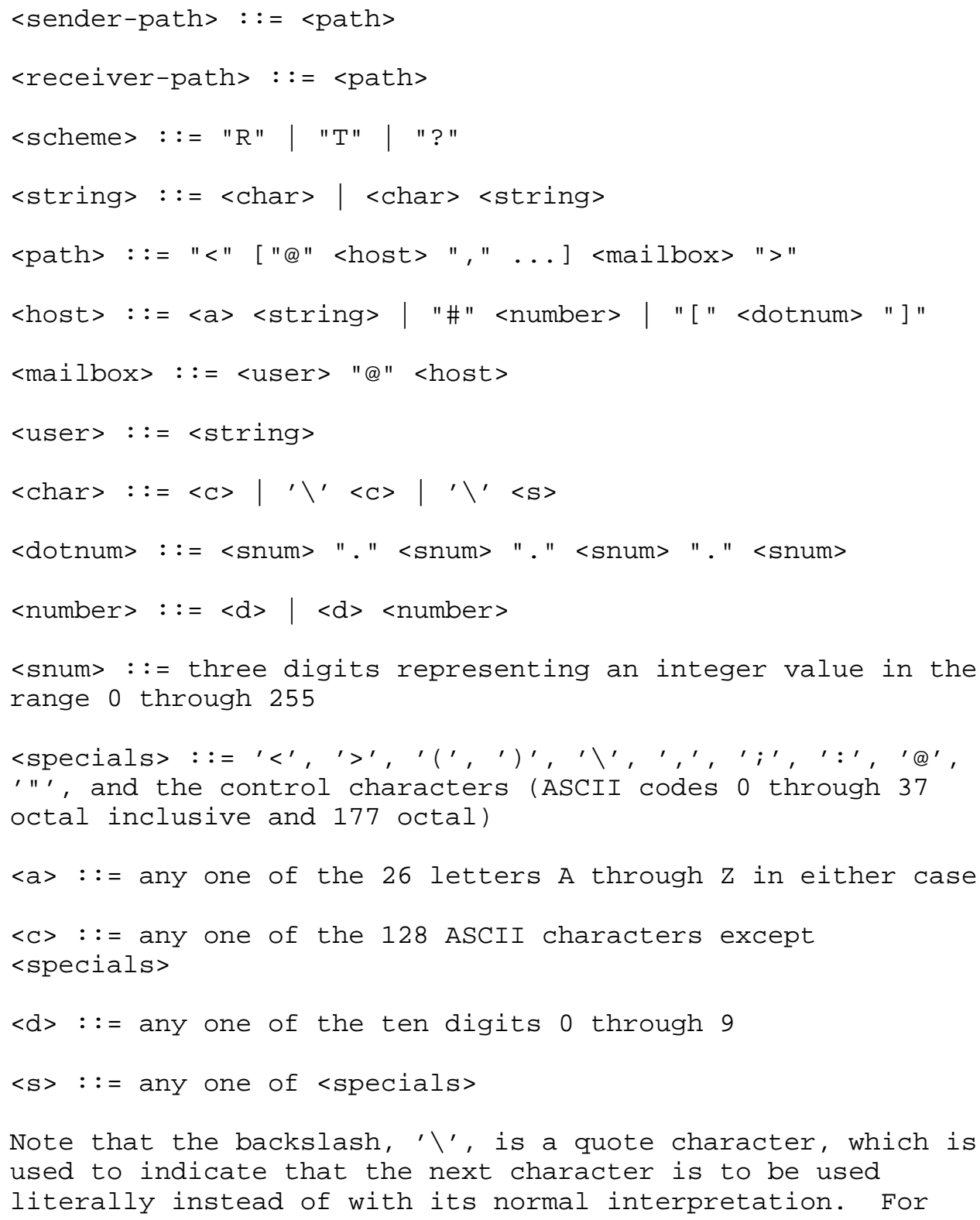




\begin{abstract}
example, "Joe\, Smith" could be used to indicate a single nine character user field with comma being the fourth character of the field.

Hosts are generally known by names which are translated to addresses in each host. Sometimes a host is not known to the translation function and communication is blocked. To bypass this barrier numeric forms are also allowed for host "names". One form is a decimal integer prefixed by a pound sign, "\#", which indicates the number is the address of the host. Another form is four small decimal integers separated by dots and enclosed by brackets, e.g., "[123.255.37.321]", which indicates a 32 bit ARPA Internet Address in four eight bit fields.
\end{abstract}




\subsection{MTP REPLIES}

Replies to MTP commands are devised to ensure the synchronization of requests and actions in the process of mail transfer, and to guarantee that the sender-MTP always knows the state of the receiver-MTP. Every command must generate exactly one reply. Additionally, some commands must occur sequentially, such as MRSQ T->MAIL->MRCP or MRSQ R->MRCP->MAIL.

The details of the command-reply sequence are made explicit in the Sections 5.3 and 5.4 on Sequencing and State Diagrams.

An MTP reply consists of a three digit number (transmitted as three alphanumeric characters) followed by some text. The number is intended for use by automata to determine what state to enter next; the text is meant for the human user. It is intended that the three digits contain enough encoded information that the sender-MTP will not need to examine the text and may either discard it or pass it on to the user, as appropriate. In particular, the text may be receiver-dependent, so there are likely to be varying texts for each reply code. Further explanation of the assignment of reply codes is given in the Appendix E on the Theory of Reply Codes. Formally, a reply is defined to be the sequence: a three-digit code, $\langle\mathrm{SP}\rangle$, one line of text, and $<\mathrm{CRLF}>$. 


\subsubsection{REPLY CODES BY FUNCTION GROUPS}

200 Command okay

201 Command okay, action aborted

500 Syntax error, command unrecognized

[This may include errors such as command line too long]

501 Syntax error in parameters or arguments

502 Command not implemented

503 Bad sequence of commands

504 Command parameter not implemented

211 System status, or system help reply

214 Help message

[Information on how to use the receiver or the meaning of a particular non-standard command; this reply is useful only to the human user]

215 <scheme> is the preferred scheme

120 <host> Service ready in nnn minutes

220 <host> Service ready for new user

221 <host> Service closing transmission channel

421 <host> Service not available, closing transmission channel [This may be a reply to any command if the service knows it must shut down]

151 User not local; will forward to <user>d<host>

152 User unknown; mail will be forwarded by the operator

250 Requested mail action okay, completed

450 Requested mail action not taken: mailbox unavailable [E.g., mailbox busy]

550 Requested action not taken: mailbox unavailable

[E.g., mailbox not found, no access]

451 Requested action aborted: local error in processing

452 Requested action not taken: insufficient system storage

552 Requested mail action aborted: exceeded storage allocation [For current mailbox location]

553 Requested action not taken: mailbox name not allowed [E.g., mailbox syntax incorrect]

354 Start mail input; end with <CRLF>. $\langle\mathrm{CRLF}>$ 


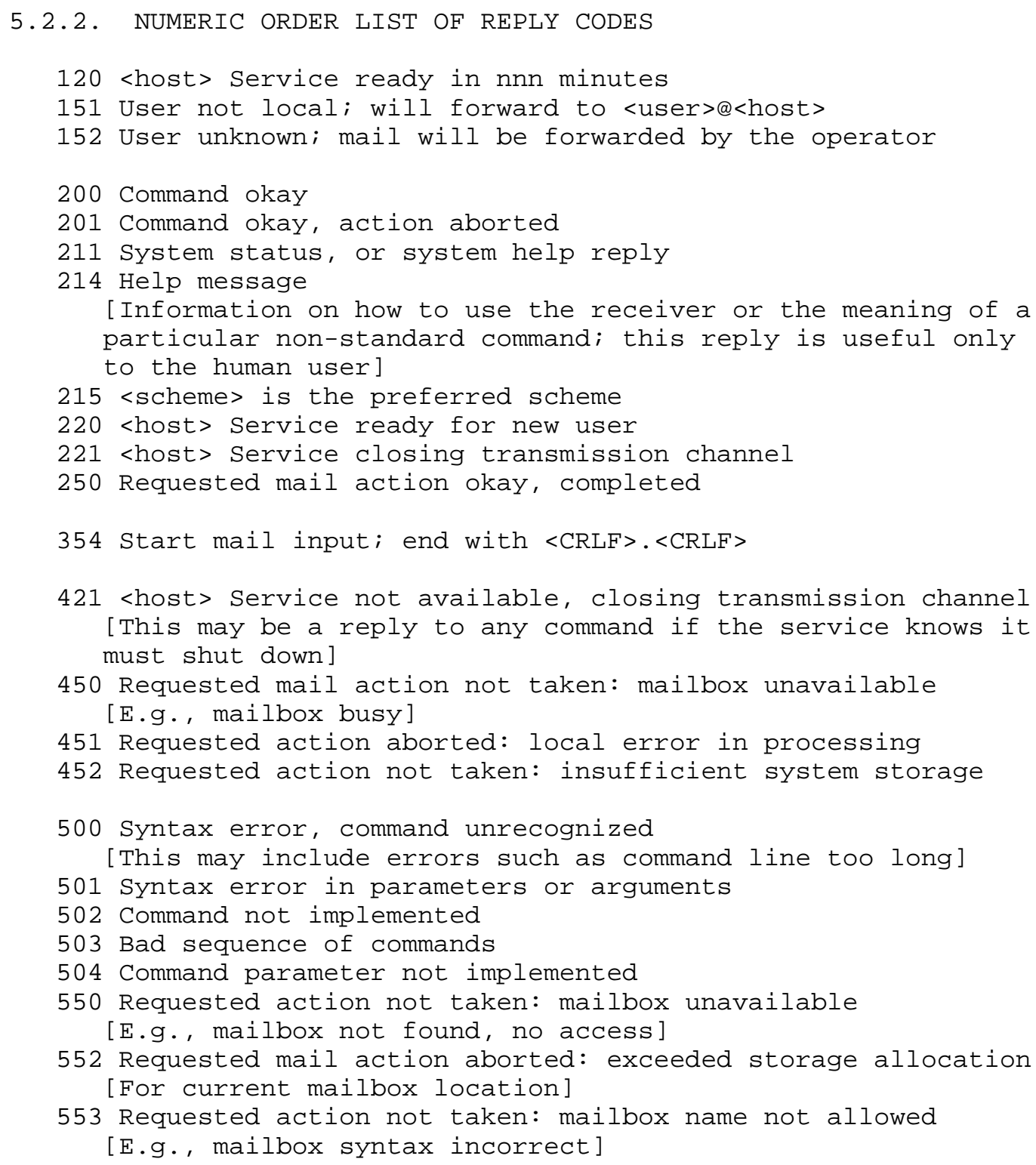




\subsection{SEQUENCING OF COMMANDS AND REPLIES}

The communication between the sender and receiver is intended to be an alternating dialogue. As such, the sender issues an MTP command and the receiver responds with a prompt primary reply. The sender should wait for this response before sending further commands.

The preliminary $(1 \mathrm{x} x)$ and intermediate (3xx) replies indicate that further commands and information are required to complete the required action. The preliminary replies require either a continue or abort command to proceed; the intermediate replies require action dependent further commands.

One important reply is the connection greetings. Under normal circumstances, a receiver will send a 220 "Awaiting input" reply when the connection is completed. The sender should wait for this greeting message before sending any commands. If the receiver is unable to accept input right away, it should send a 120 "Expected delay" reply immediately. The sender can then indicate it is willing to wait via a continue command, or not via the abort command. The receiver will respond to the abort with a 201 reply, and to the continue with the 220 reply when ready.

Note: all the greeting type replies have the official name of the server host as the first word following the reply code.

For example,

$$
220<\mathrm{SP}>\mathrm{USC}-\mathrm{ISIF}<\mathrm{SP}>\text { Service ready }<\mathrm{CRLF}>
$$

The table below lists alternative success and failure replies for each command. These must be strictly adhered to; a receiver may substitute text in the replies, but the meaning and action implied by the code numbers and by the specific command reply sequence cannot be altered.

COMMAND-REPLY SEQUENCES

Each command is listed with its possible replies. Preliminary replies are listed first with their succeeding replies indented under them, then success and failure completion, and finally intermediary replies with the remaining commands from the sequence following. The prefixes used before the possible replies are "P" for preliminary, "I" for intermediate, "S" for success, "F" for failure, and "E" for error. The 421 reply 
May 1981

Mail Transfer Protocol

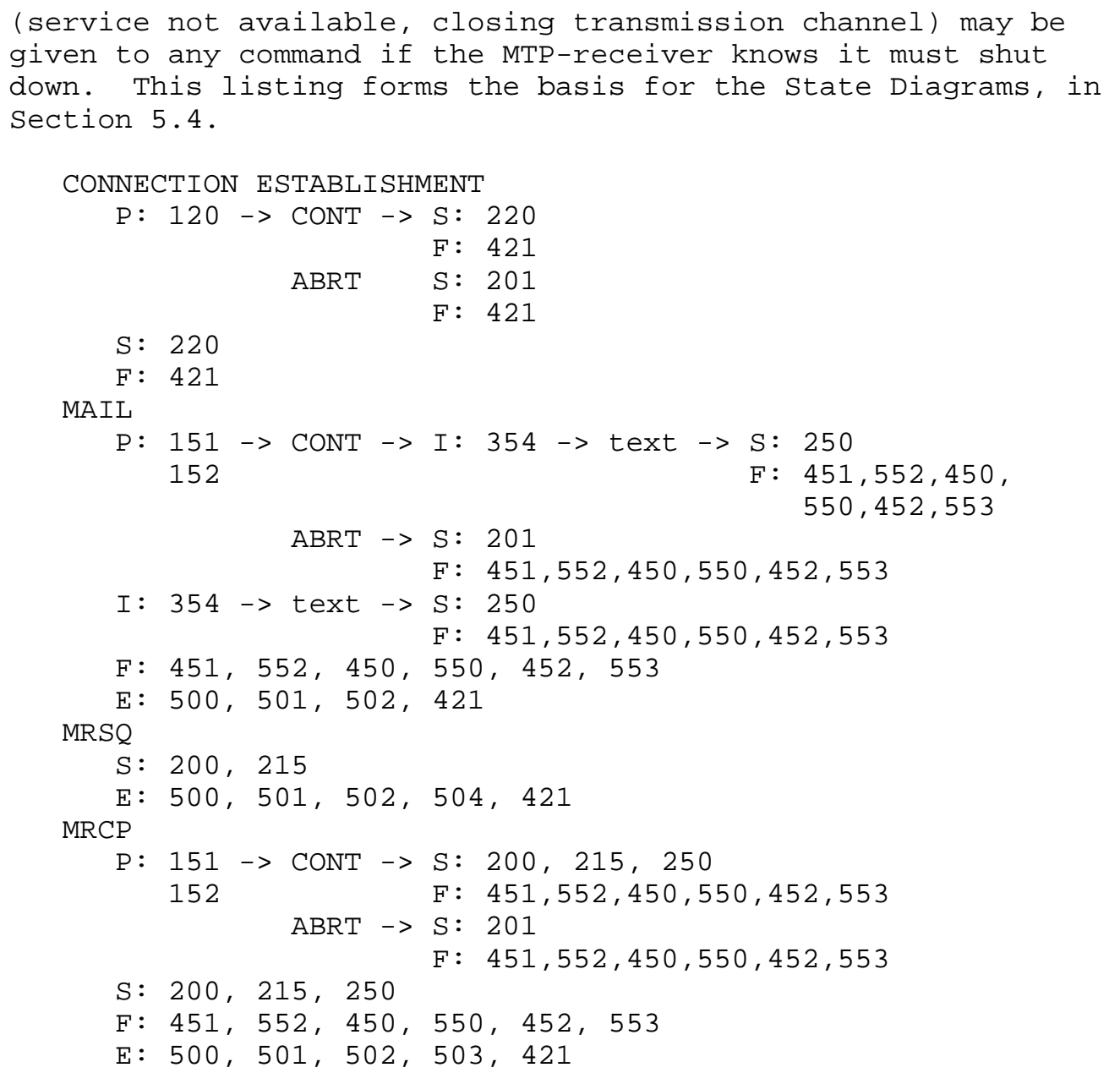




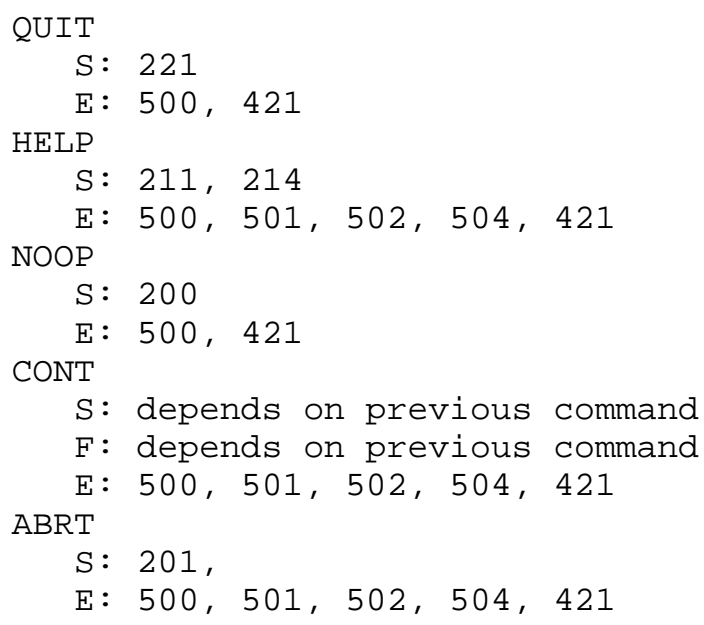




\subsection{STATE DIAGRAMS}

Following are state diagrams for a very simple minded MTP implementation. Only the first digit of the reply codes is used. There is one state diagram for each group of MTP commands.

The command groupings were determined by constructing a model for each command and then collecting together the commands with structurally identical models.

For each command there are three possible outcomes: "success" $(S)$, "failure" (F), and "error" (E). In the state diagrams below we use the symbol B for "begin", and the symbol W for "wait for reply".

First, the diagram that represents most of the MTP commands:

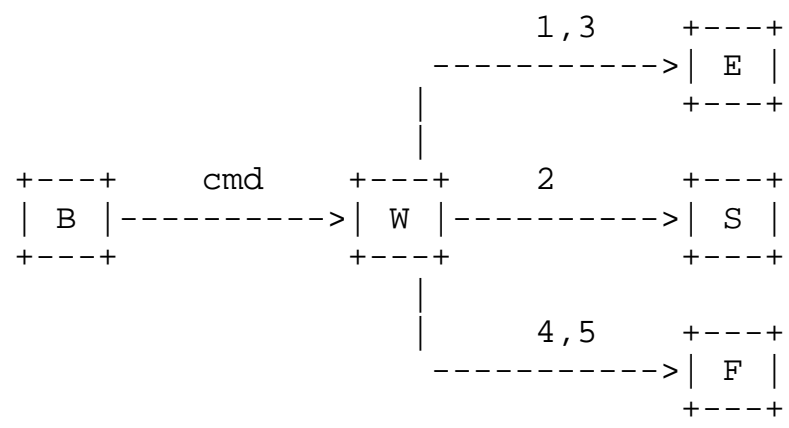

This diagram models the commands:

HELP, MRCP, MRSQ, NOOP, QUIT, ABRT. 
A more complex diagram models the MAIL command:

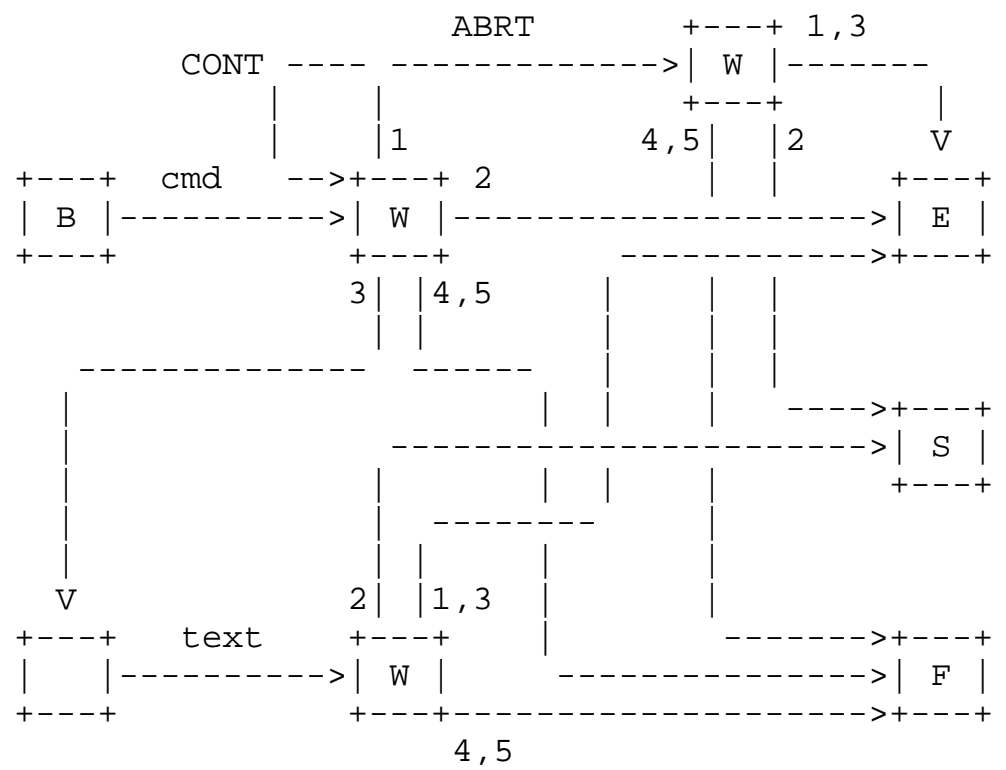

Note that the "text" here is a series of lines sent from the sender to the receiver with no response expected until the last line is sent. 


\subsection{DETAILS}

\subsubsection{MINIMUM IMPLEMENTATION}

In order to make MTP workable, the following minimum implementation is required for all receivers:

$$
\begin{array}{r}
\text { COMMANDS -- } \\
\text { QUIL } \\
\text { NOIT } \\
\text { NOOP }
\end{array}
$$

\subsubsection{TRANSPARENCY}

Without some provision for data transparency the character sequence " $\angle \mathrm{CRLF}>$. $<\mathrm{CRLF}>$ " ends the the mail text and cannot be sent by the user. In general, users are not aware of such "forbidden" sequences. To allow all user composed text to be transmitted transparently the following procedures are used.

1. Before sending a line of mail text the sender-MTP checks the first character of the line. If it is a period, one additional period is inserted at the beginning of the line.

2. When a line of mail text is received by the receiver-MTP it checks the the line. If the line is composed of a single period it is the end of mail. If the first character is a period and there are other characters on the line, the first character is deleted.

5.5.3. SIZES

There are several objects that ought to have defined maximum sizes.

$$
\text { user }
$$

The maximum total length of a user name is 40 characters. host

The maximum total length of a host name or number is 20 characters. 
path

The maximum total length of a sender-path or receiver-path is 100 characters.

command line

The maximum total length of a command line including the command word and the $\langle\mathrm{CRLF}\rangle$ is 200 characters.

reply line

The maximum total length of a reply line including the reply code and the $\langle\mathrm{CRLF}>$ is 65 characters.

text line

The maximum total length of a text line including the the $<$ CRLF $>$ is 1000 characters.

To the maximum extent possible implementation techniques which impose no limits at all to the length of these objects should be used. 
May 1981

Mail Transfer Protocol

\section{APPENDIX A}

TCP Transport service

The Transmission Control Protocol [1] is used in the ARPA

Internet, and in any network following the US DoD standards for internetwork protocols.

Connection Establishment

The MTP transmission channel is a TCP connection established between the sender process port $U$ and the receiver process port L. This single full duplex connection is used as the transmission channel. This protocol is assigned the service port 57 (71 octal), that is $\mathrm{L}=57$.

Data Transfer

The TCP connection supports the transmission of 8-bit bytes. The MTP data is 7-bit ASCII characters. Each character is transmitted as a 8-bit byte with the high-order bit cleared to zero. 


\section{APPENDIX B}

NCP Transport service

The ARPANET Host-to-Host Protocol [2] (implemented by the Network Control Program) may be used in the ARPANET.

Connection Establishment

The MTP transmission channel is established via NCP between the the sender process socket $U$ and receiver process socket $L$. The Initial Connection Protocol [3] is followed resulting in a pair of simplex connections. This pair of connections is used as the transmission channel. This protocol is assigned the contact socket 57 (71 octal), that is $\mathrm{L}=57$.

Data Transfer

The NCP data connections are established in 8-bit byte mode. The MTP data is 7-bit ASCII characters. Each character is transmitted as a 8-bit byte with the high-order bit cleared to zero. 
May 1981

Mail Transfer Protocol

APPENDIX C

NITS

The Network Independent Transport Service [4] may be used.

Connection Establishment

The MTP transmission channel is established via NITS between

the the sender process and receiver process. The sender

process executes the CONNECT primitive, and the waiting

receiver process executes the ACCEPT primitive.

Data Transfer

The NITS connection supports the transmission of 8-bit bytes . The MTP data is 7-bit ASCII characters. Each character is transmitted as a 8-bit byte with the high-order bit cleared to zero. 


\section{APPENDIX D}

X.25 Transport service

It may be possible to use the $\mathrm{x} .25$ service [5] as provided by the Public Data Networks directly, but there are indications that it is too error prone to qualify as a reliable channel. It is suggested that a reliable end-to-end protocol such as TCP be used on top of X.25 connections. 


\section{APPENDIX E}

Theory of Reply Codes

The three digits of the reply each have a special significance. The first digit denotes whether the response is good, bad or incomplete. An unsophisticated sender-MTP will be able to determine its next action (proceed as planned, redo, retrench, etc.) by simply examining this first digit. A sender-MTP that wants to know approximately what kind of error occurred (e.g., mail system error, command syntax error) may examine the second digit, reserving the third digit for the finest gradation of information.

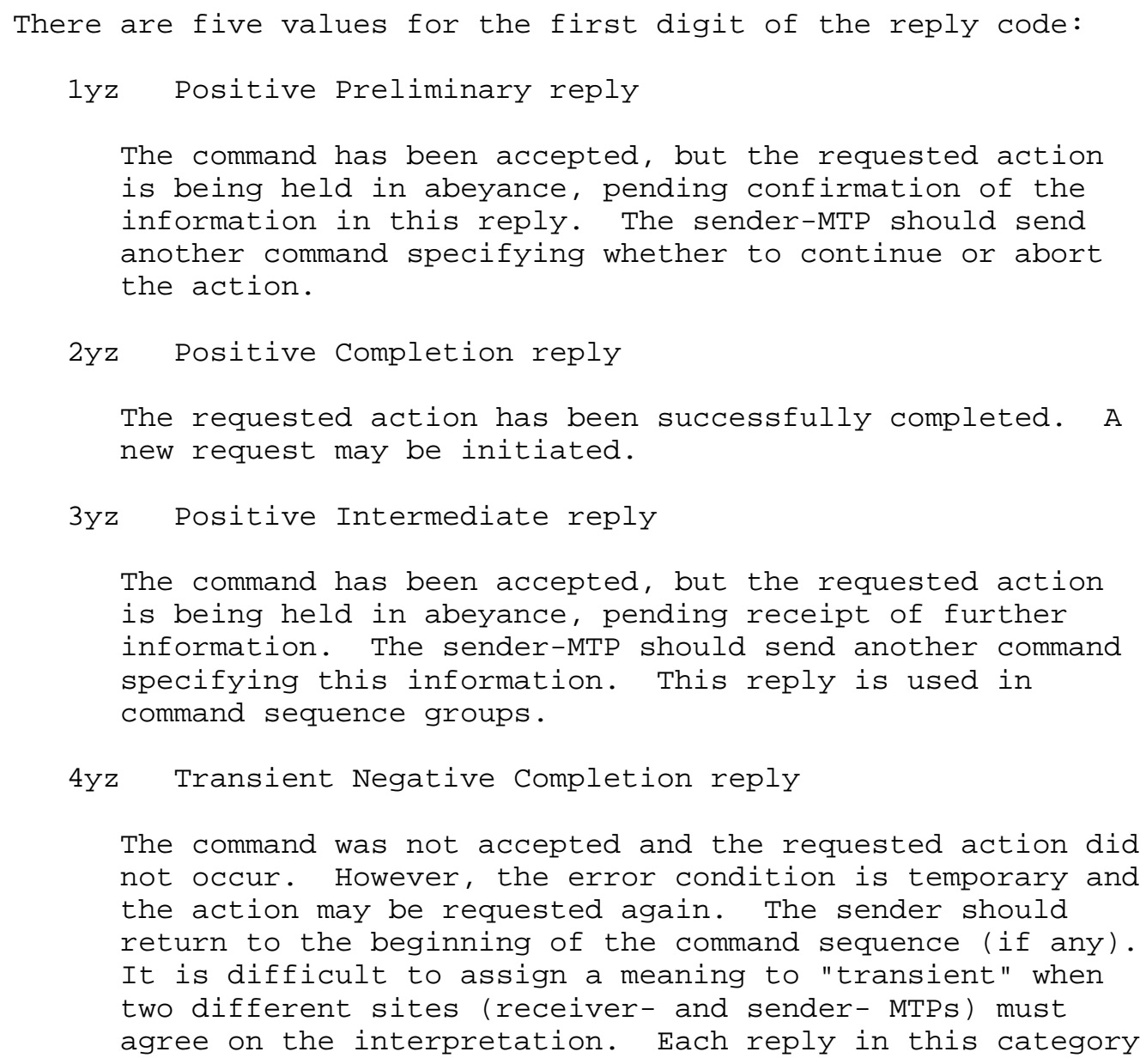




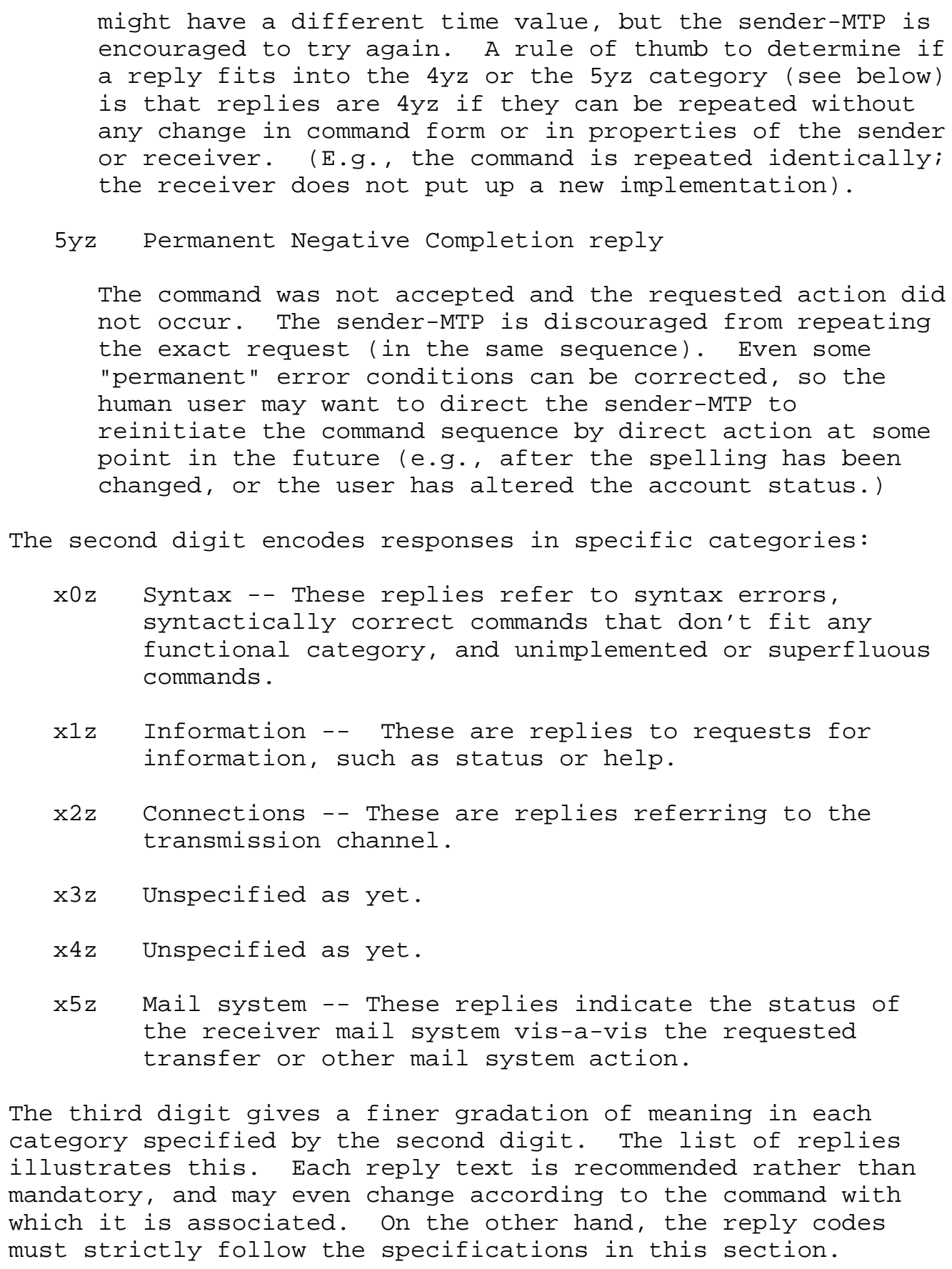


Receiver implementations should not invent new codes for slightly different situations from the ones described here, but rather adapt codes already defined.

For example, a command such as NOOP whose successful execution does not offer the sender-MTP any new information will return a 200 reply. The response is 502 when the command requests an unimplemented non-site-specific action. A refinement of that is the 504 reply for a command that is implemented, but that requests an unimplemented parameter.

The reply text may be longer than a single line; in these cases the complete text must be marked so the sender-MTP knows when it can stop reading the reply. This requires a special format to indicate a multiple line reply.

The format for multi-line replies requires that every line, except the last, begin with the reply code, followed immediately by a hyphen, "-" (also known as minus), followed by text. The last line will begin with the reply code, followed immediately by $\langle\mathrm{SP}\rangle$, optionally some text, and $<\mathrm{CRLF}\rangle$.

For example:

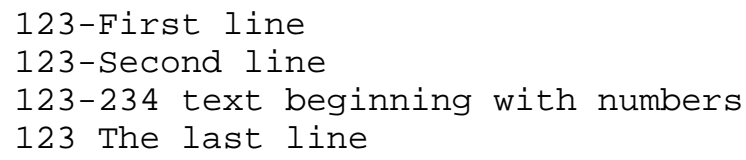

The sender-MTP then simply needs to search for the reply code followed by <SP> at the beginning of a line, and ignore all preceding lines. 
GLOSSARY

ASCII

American Standard Code for Information Interchange [6]. command

A request for a mail service action sent by the sender-MTP to the receiver-MTP.

host

A computer in the internetwork environment on which mailboxes or MTP processes reside.

line

A line of text ending with a $<$ CRLF $>$.

mail

A sequence of ASCII characters of arbitrary length, which conforms to the standard set in RFC 733 (Standard for the Format of ARPA Network Text Messages [7]).

mailbox

A character string (address) which identifies a user to whom mail is to be sent. Mailbox normally consists of the host and user specifications. The standard mailbox naming convention is defined to be "user@host". Additionally, the "container" in which mail is stored.

receiver-MTP process

A process which transfers mail in cooperation with a sender-MTP process. It waits for a connection to be established via the transport service. It receives MTP commands from the sender-MTP, sends replies, and governs the transfer of mail. 
reply

A reply is an acknowledgment (positive or negative) sent from receiver to sender via the transmission channel in response to a MTP command. The general form of a reply is a completion code (including error codes) followed by a text string. The codes are for use by programs and the text is usually intended for human users.

sender-MTP process

A process which transfers mail in cooperation with a receiver-MTP process. A local language may be used in the user interface command/reply dialogue. The sender-MTP initiates the transport service connection. It initiates MTP commands, receives replies, and governs the transfer of mail.

transmission channel

A full-duplex communication path between a sender-MTP and a receiver-MTP for the exchange of commands, replies, and mail text.

transport service

Any reliable stream-oriented data communication services. For example, NCP, TCP, NITS.

user

A human being (or a process on behalf of a human being) wishing to obtain mail transfer service. In addition, a recipient of computer mail.

word

A human being (or a process on behalf of a human being) wishing to obtain mail transfer service. In addition, a recipient of computer mail.

$<\mathrm{CRLF}>$

The characters carriage return and line feed (in that order). 
May 1981

Mail Transfer Protocol

$<\mathrm{SP}>$

The space character. 


\section{REFERENCES}

\section{[1] $\mathrm{TCP}$}

Postel, J., ed., "DOD Standard Transmission Control Protocol", IEN 129, RFC 761, USC/Information Sciences Institute, NTIS ADA082609, January 1980. Appears in: Computer Communication Review, Special Interest Group on Data Communications, ACM, V.10, N.4, October 1980 .

[2] $\mathrm{NCP}$

McKenzie,A., "Host/Host Protocol for the ARPA Network", NIC 8246, January 1972. Also in: Feinler, E. and J. Postel, eds., "ARPANET Protocol Handbook", NIC 7104, for the Defense Communications Agency by SRI International, Menlo Park, California, Revised January 1978 .

[3] Initial Connection Protocol

Postel, J., "Official Initial Connection Protocol", NIC 7101, 11 June 1971. Also in: Feinler, E. and J. Postel, eds., "ARPANET Protocol Handbook", NIC 7104, for the Defense Communications Agency by SRI International, Menlo Park, California, Revised January 1978 .

[4] NITS

PSS/SG3, "A Network Independent Transport Service", Study Group 3, The Post Office PSS Users Group, February 1980. Available from the DCPU, National Physical Laboratory, Teddington, UK.

\section{[5] X.25}

CCITT, "Recommendation X.25 - Interface Between Data Terminal Equipment (DTE) and Data Circuit-terminating Equipment (DCE) for Terminals Operating in the Packet Mode on Public Data Networks," CCITT Orange Book, Vol. VIII.2, International Telephone and Telegraph Consultative Committee, Geneva, 1976. 
[6] ASCII

ASCII, "USA Code for Information Interchange", United States of America Standards Institute, X3.4, 1968. Also in: Feinler, E. and J. Postel, eds., "ARPANET Protocol Handbook", NIC 7104, for the Defense Communications Agency by SRI International, Menlo Park, California, Revised January 1978.

[7] $\quad \mathrm{RFC} 733$

Crocker, D., J. Vittal, K. Pogran, and D. Henderson, "Standard for the Format of ARPA Network Text Messages," RFC 733, NIC 41952, November 1977. Also in: Feinler, E. and J. Postel, eds., "ARPANET Protocol Handbook", NIC 7104, for the Defense Communications Agency by SRI International, Menlo Park, California, Revised January 1978. 